\title{
PAINTING SELF-CONSISTENT BEAM DISTRIBUTIONS IN RINGS
}

\author{
J. A. Holmes, V. V. Danilov, S. M. Cousineau, SNS, ORNL, Oak Ridge, TN 37831, U.S.A.
}

\section{Abstract}

We define self-consistent beam distributions to have the following properties: 1) time-independence or periodicity, 2) linear space charge forces, and 3) maintainance of their defining shape and density under all linear transformations. The periodic condition guarantees zero space-charge-induced halo growth and beam loss during injection. Some self-consistent distributions can be manipulated into flat, or possibly even point-like, beams, which makes them interesting to colliders and to heavyion fusion. This paper discusses methods for painting 2D and 3D self-consistent distributions and for their manipulation to produce flat and point-like beams.

\section{INTRODUCTION}

In previous papers [1-3], we described and studied a set of beam distributions which we call self-consistent. By self-consistent, we mean beam distributions that 1) are time-independent or periodic in time, 2) support linear space charge forces, and 3) maintain their defining shape and density under all linear transformations. Reference 1 generalized previous studies of self-consistent beams [49] by demonstrating a systematic method to create an infinite number of self-consistent distributions in any number of dimensions. In phase space these distributions are functions of the constants of motion that take the form of functions of the Hamiltonian times $\delta$ functions of linear combinations of the phase space variables. The distributions are classified by the notation $\{\mathrm{n}, \mathrm{m}\}$, where $\mathrm{n}$ is the number of spatial dimensions and $m$ is the number of $\delta$ functions of linear phase space combinations. These distributions are characterized by ellipsoidal or linear relationships between the phase space variables and by constant particle densities. Self-consistent distributions are of interest in high intensity accelerators because they have the desirable property of suppressing space-chargeinduced halo growth and associated beam loss. Furthermore, certain self-consistent distributions can be manipulated to produce flat, or possibly even point-like, beams, and are therefore candidates for applications in colliders and heavy ion fusion. References [2,3] studied some of the properties of these distributions. In this paper we illustrate certain features of self-consistent distributions. Specifically, we demonstrate the painting of a 2D self-consistent coasting beam into the SNS ring and the use of solenoids to maintain self-consistency in the presence of nonlinear forces. We also show the transport of a 3D self-consistent distribution followed by its compression to a flat beam through the use of a skew quadrupole and subsequent transport.

\section{PAINTING A SELF-CONSISTENT COASTING BEAM INTO SNS}

In Ref. 1, we demonstrated the painting of a selfconsistent coasting beam distribution of type $\{2,2\}$ into the SNS lattice. This was accomplished by setting the lattice tunes equal and by painting in $x^{\prime}$ and $y^{\prime}$ as well as in $\mathrm{x}$ and $\mathrm{y}$. The necessary injection bump waveforms are within the capability of the SNS injection kickers. The calculation was carried out neglecting space charge and assuming purely linear transport. Subsequent calculations demonstrate that even small nonlinearities, such as quadrupole fringe fields or activation of chromaticity correction sextupoles, destroys the self-consistency: ellipticity is lost along with the uniform density. If, in addition to these nonlinearities the space charge force is introduced, the beam distribution again becomes elliptical, although the ellipse has a different shape in response to the space-charge-induced change in the beta functions.

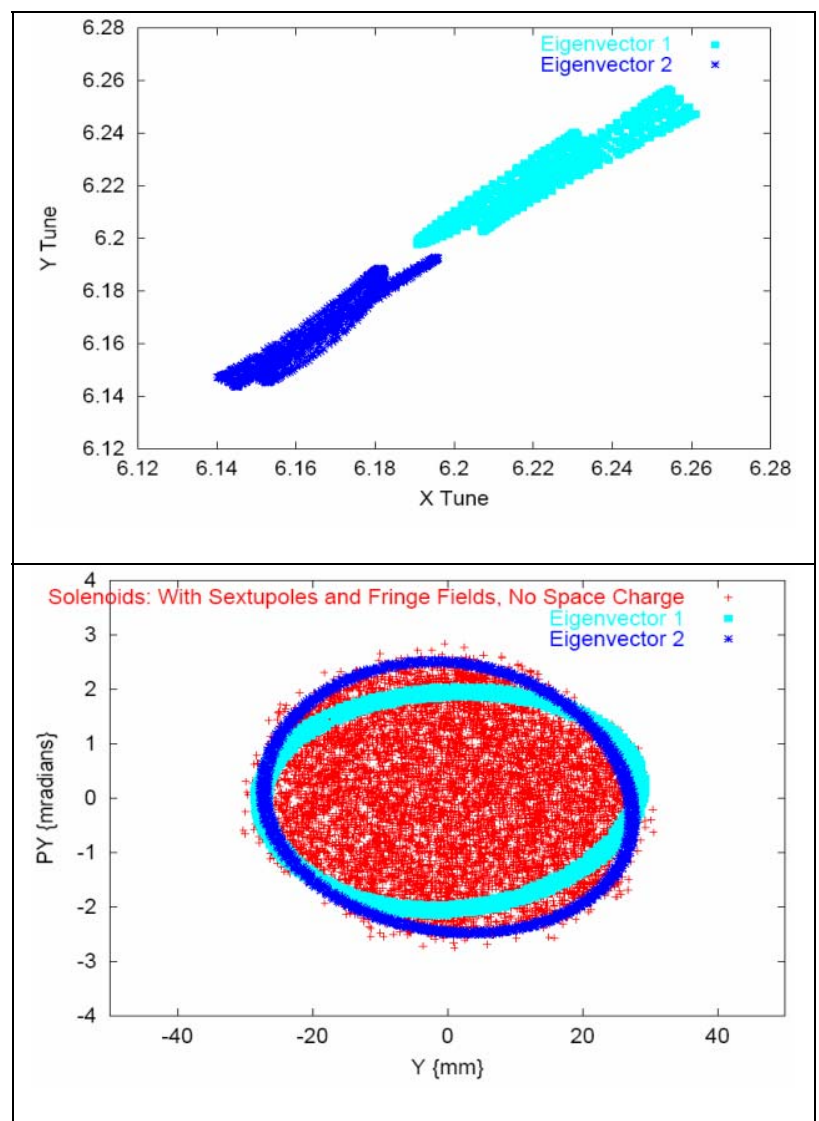

Figure 1: Top) Tune footprint for the two eigenvectors, each taken turn by turn over 1000 turns. Bottom) Eigenvectors in vertical phase space, together with self-consistent beam distribution painted to one of the eigenvectors. 
In order to make the self-consistency more robust in the presence of nonlinearities, we introduce two solenoids symmetrically into the SNS lattice and activate them in order to split the lattice tunes (Fig. 1). The presence of the solenoids leads to two pairs of complex conjugate eigenfunctions with conjugate eigenvalues for the ring transfer matrix in transverse phase space, and it is possible to paint a self-consistent $\{2,2\}$ distribution to the real or imaginary part of either of the eigenfunction pairs (Fig. 1). As in the previous case, the painting involves $x^{\prime}$ and $y^{\prime}$ as well as $x$ and $y$ and is within the capabilities of the SNS injection kickers. The results show that a selfconsistent elliptical beam of uniform charge density is obtained, even in the presence of fringe fields and chromaticity correction sextupoles. The addition of space charge leads to results that are similar to those obtained without solenoids, in the sense that the ellipse has a different shape and the particle distribution is less uniform.

Although we have succeeded in painting selfconsistent 2D distributions, we have not yet done so for a 3D distribution. Maintaining self-consistency during painting for a $2 \mathrm{D}$ distribution is relatively straightforward for a machine like SNS. The time scale for painting (1000 turns in SNS) is very slow compared to betatron oscillations (six per turn in SNS), so for irrational betatron tunes the beam is always well mixed right out to the most recently painted edges. Thus, a self-consistent distribution can be easily maintained throughout the painting process. To maintain a self-consistent 3D beam throughout painting will require laying on spherical shells with all the mathematical self-consistency relationships. This requires a time scale for painting of less than 1 turn, rather than 1000 turns. Also, 3D self-consistency will require variation of the energy, as well as the transverse phase space coordinates, during every turn, and this variation must increase throughout the accumulation process.

\section{MAKING A FLAT BEAM FROM A 3D SELF-CONSISTENT DISTRIBUTION}

As a further study, we created a flat beam from a $\{3,2\}$ distribution. Such a distribution is a 3D constant density ellipsoid in all projections except for two linear relationships, which we chose between $x-y^{\prime}$ and between $y-x^{\prime}$, as shown in Fig. 2. Although we have not yet tried to paint such a distribution, we defined one numerically with parameters matching it into the SNS lattice. To test the self-consistency we transported this distribution linearly without change for 100 turns assuming no space charge (Fig. 2).

After 100 turns, we passed the beam through a skew quadrupole with strength chosen to zero out $y^{\prime}$, as shown in Fig. 3. This is possible because of the linear relationship between $\mathrm{x}$ and $\mathrm{y}^{\prime}$ given by the $\{3,2\}$ distribution. Following the skew quadrupole, the beam is transported for a $\pi / 2$ betatron phase advance so that, rather than $y^{\prime}$, it is now y that is zero, giving a flat beam.

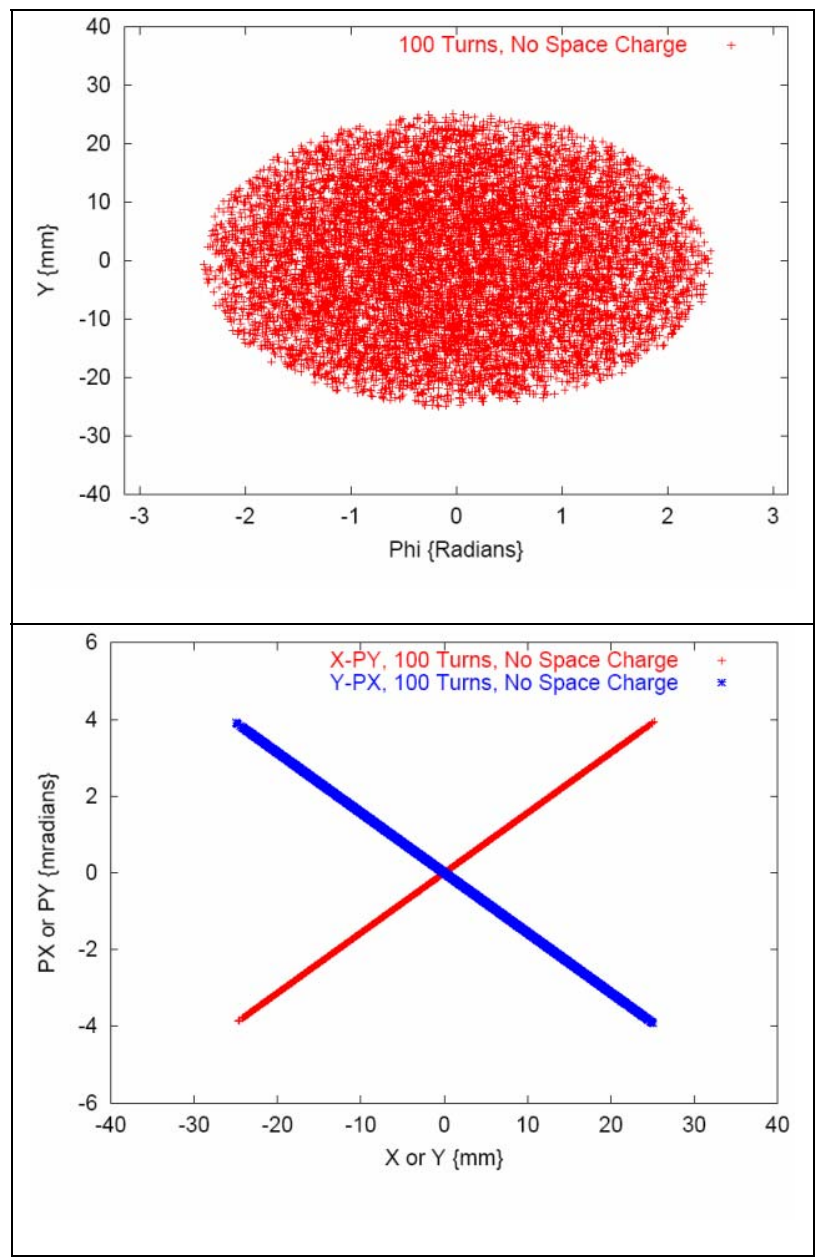

Figure 2: $\quad$ Top) $y-\varphi$ projection for matched $\{3,2\}$ distribution after 100 turns. Bottom) $x-y^{\prime}$ and $y-x^{\prime}$ projections for matched $\{3,2\}$ distribution after 100 turns.

Unlike the coasting beam studies shown above, this work was carried out using linear transport and neglecting space charge. Neglecting space charge is justified for high energy beams, as in colliders, where intrabeam space charge forces are small. Future studies will focus on the effects of nonlinearities as well as on methods to overcome the difficulties of painting 3D self-consistent distributions that were discussed above.

Another possibility involves the use of self-consistent distributions for the production of point-like beams. This is not possible using a skew quadrupole for the $\{3,2\}$ distribution here because the $x-y^{\prime}$ and $y-x^{\prime}$ relations shown in Fig. 2 are not aligned. We have found that for half integer betatron tunes, the scheme we use to paint $\{2,2\}$ distributions leads to linear relationships between $\mathrm{x}-\mathrm{x}^{\prime}$ and $y-y^{\prime}$ as well as $x-y^{\prime}$ and $y-x^{\prime}$. In this case, if the tunes are equal and a normal quadrupole is placed at a location where the beta functions are equal, the quadrupole strength can be used to zero $x^{\prime}$ and $y^{\prime}$ simultaneously. Then, a $\pi / 2$ betatron phase advance will lead to a point beam. Unfortunately, the painting scheme does not produce a self-consistent beam for rational tunes and nonlinear effects will lead to additional problems. We are 
studying other possibilities for creating self-consistent beams that can be manipulated to give point beams. Clearly, we will need at least two linear relationships between phase space coordinates to accomplish this.

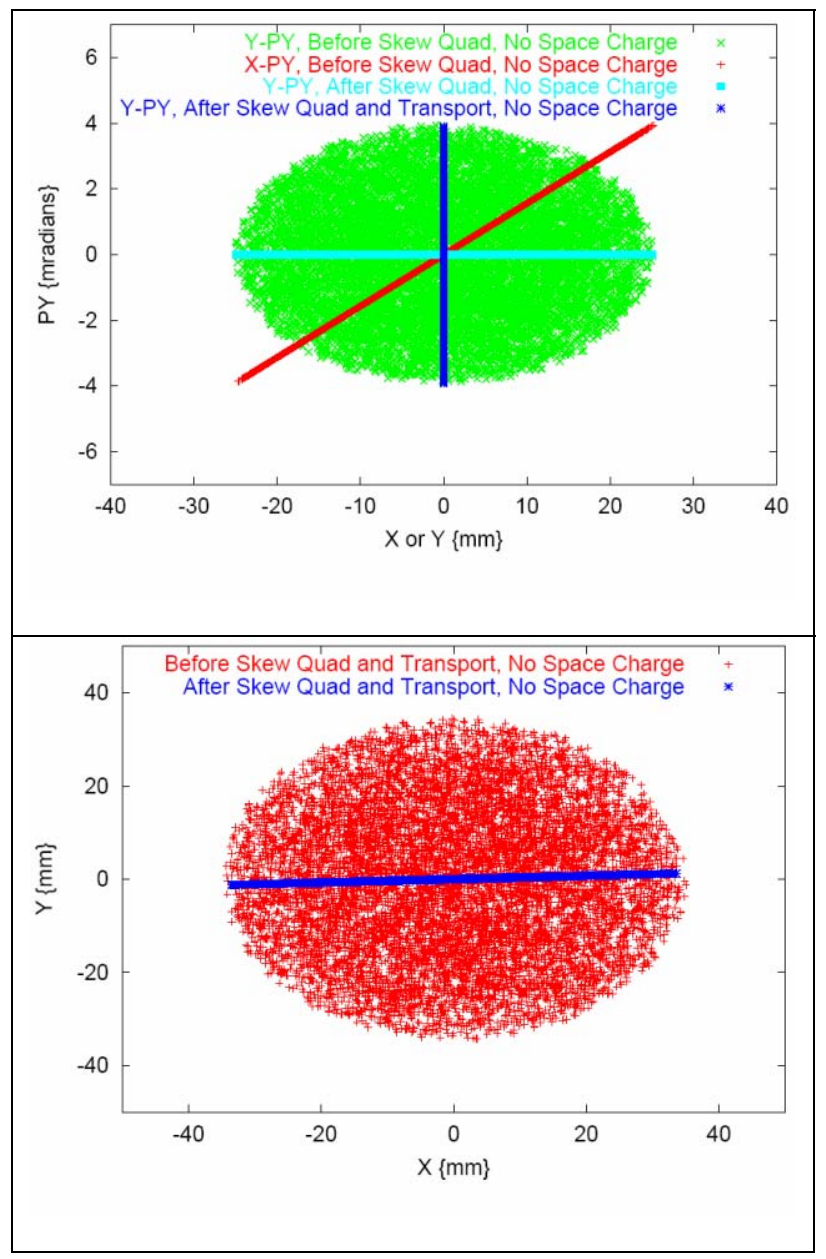

Figure 3: Top) Creation of flat beam by passing $\{3,2\}$ distribution through skew quadrupole followed by transport. Bottom) Real space transverse projections of beam before and after flattening procedure.

\section{ACKNOWLEDGMENT}

SNS is managed by UT-Battelle, LLC, under contract DE-AC05-00OR22725 for the U.S. Department of Energy. SNS is a partnership of six national laboratories:
Argonne, Brookhaven, Jefferson, Lawrence Berkeley, Los Alamos and Oak Ridge.

\section{REFERENCES}

1. Danilov, V., Cousineau, S., Henderson, S., and Holmes, J., Phys. Rev. ST Accel. Beams 6, 094202 (2003).

2. Danilov, V., Cousineau, S., Henderson, S., Holmes, J., and Plum, M., "Injection Schemes for Self-Consistent Space Charge Distributions", in Proceedings of the European Particle Accelerator Conference (EPAC 2004), Lucerne, 2004.

3. J. Holmes, Danilov, V., Cousineau, S., Henderson, S., and Plum, M., "Painting Self-Consistent Beam Distributions in Rings", in Proceedings of the $33^{\text {rd }}$ ICFA Advanced Beam Dynamics Workshop, Bensheim/Darmstadt, (2004).

4. I.M. Kapchinsky and V.V. Vladimirsky, "Limitations of Proton Beam Current in a Strong Focusing Linear Accelerator with the Beam Space Charge", in Proceedings of the Conference on High Energy Accelerators and Instrumentation, CERN, 1959, p. 274.

5. F.J. Sacherer, "Transverse Space Charge Effects in Circular Accelerators", Ph.D. Thesis, LBNL, Berkeley, CA, October, 1968.

6. Yu. K. Batygin, "Adiabatic Matching of a Nonuniform Intense Charged-Particle Beam into the Focusing Channel", Phys. Rev. E, 54, 5 (1996) pp 5673-5680.

7. R.L. Glukstern, A.V. Fedotov, S.S. Kurennoy, R.D. Ryne, "Longitudinal Halo in Beam Bunches with SelfConsistent 6-D Distributions", in Proceedings of Workshop on Space Charge Physics in High Intensity Hadron Rings, Shelter Island NY (1998) (AIP Conference Proceedings 448, Woodbury, NY, 1998).

8. R.C. Davidson, H. Qin, S.I. Tsenov, and E.A. Startsev, " Kinetic Description of Intense Beam Propogation Through a Periodic Focusing Field for Uniform PhaseSpace Density", Phys. Rev. ST Accel. Beams 5, $084402(2002)$.

9. V. V. Danilov and E.A. Perevedentsev, "Two Examples of Integrable Systems with Round Colliding Beams", Proceedings of the Particle Accelerator Conference, Vancouver, 1997 (IEEE, Piscataway, NJ, 1998), p. 1759. 